Applied Mathematical Sciences, Vol. 15, 2021, no. 11, 505 - 512

HIKARI Ltd, www.m-hikari.com

https://doi.org/10.12988/ams.2021.914551

\title{
On Retrospective Insurance Premium
}

\author{
Antonella Campana \\ Department of Economics \\ University of Molise \\ Campobasso, Italy \\ Paola Ferretti \\ Department of Economics \\ Ca' Foscari University of Venice \\ Venice, Italy
}

This article is distributed under the Creative Commons by-nc-nd Attribution License. Copyright (C) 2021 Hikari Ltd.

\begin{abstract}
In this paper we focus on the expected retrospective premium under equivalent representations. Some general properties related to extreme premia (minumum and maximum) are presented. The particular case of a risk with translated gamma distribution is discussed.
\end{abstract}

\section{Mathematics Subject Classification: 62P05}

Keywords: retrospective rating plan, expected retrospective premium, stochastic premium, non-proportional reinsurance

\section{Introduction}

In insurance practice, premiums with a random component are often applied. As an example, in reinsurance contracts, when the reinsurer makes a profit above a certain threshold, he generally pays the insurer a commission on the profit. In this way, the effective cost of the reinsurance coverage of the direct insurer has a random component: given the fixed ex ante reinsurance premium, also the reimbursements received by the reinsurer have to be taken into account. Another example of a random insurance and reinsurance premium 
is that expressed under the retrospective premium principle. This calculation method is widely used in liability insurance and worker compensation in the United States.

Retrospectively rated insurance is an insurance policy with a premium that adjusts according to the losses experienced by the insured company, rather than according to industry-wide loss experience. An initial premium is charged and adjustments are performed periodically after the policy has expired. This method serves as an incentive to the insured company to control its losses since the price of the policy is likely to decrease if the insured is able to limit risk exposure. The premium can be adjusted within a certain range of values, and it is subject to a minimum and a maximum amount.

In actuarial literature not so many papers analized this widespread practice from the analytical point of view (see $[3,5,7,8,9]$ ). In this paper, after a description of the retrospective rating plan (Section 2), we focus on the expected retrospective premium (Section 3) and, through some equivalent representations, we are able to prove some of its properties. The Section 4 analyzes the expected retrospective premium in the particular case of a risk with translated gamma distribution.

Section 5 contains some observations and research suggestions.

\section{Retrospective rating plan}

With the aim of taking into account the insured's current experience of loss, the insurance practice proposes a rating plan in which the premium can be adjusted, but with the condition that it still remains between a minimum and a maximum value. Among the different literature proposals (see $[3,8]$ ), we make the choice of referring to the following definition of retrospective premium for a non-negative risk $Y$, with $E[Y]<\infty$ :

$$
\Pi(Y)=\min \left\{\max \left\{\left(B_{Y}+L Y\right) T, G_{Y}\right\}, H_{Y}\right\}
$$

where:

$L \geq 0$ is the loss conversion factor that covers the loss adjustment expenses;

$T>1$ is the tax multiplier including premium tax;

$B_{Y}, G_{Y}$ and $H_{Y}$ are respectively, the basic, the minimum and the maximum premium, all are non-negative.

In literature, $B_{Y}, G_{Y}, H_{Y}, L$ and $T$ are called rating parameters; they are related to each other, in fact by definition (1) it is $G_{Y} \leq \Pi(Y) \leq H_{Y}$.

To avoid the case of a deterministic premium $\Pi(Y)$, from now on it is assumed $G_{Y}<H_{Y}$ and $L>0$. 
The basic premium is generally obtained as the minimum amount $B_{Y}$ such that the expected retrospective premium $E[\Pi(Y)]$ assumes a not negative fixed value $K$ not exceeding the maximum premium $H_{Y}: E[\Pi(Y)]=K$ where $0 \leq K \leq H_{Y}$.

In the recent paper [3], the Authors consider the retrospective premium as a reinsurance premium and set some hypothesis on the rating parameters: they assume $G_{Y}=0, H_{Y}=(1+\theta) E[Y]$, and they set the basic premium $B_{Y}$ equal to the minimal solution of the following equation: $E[\Pi(Y)]=(1+k) E[Y]$, $(0<k<\theta)$. The insurers total risk exposure is shown to be increasing in parameters $L$ and $\theta$ in the sense of convex order (Theorem 3.1 in [3]).

In this paper we consider a different formalization of the retrospective premium $\Pi(Y)$ by referring to $y_{m}$ and $y_{M}$ where $y_{m} \leq y_{M}$. In this way, $\Pi(Y)$ is defined as:

$$
\Pi(Y)=\left\{\begin{array}{ll}
G_{Y} & \text { for } Y \leq y_{m} \\
\left(B_{Y}+L Y\right) T & \text { for } y_{m}<Y<y_{M} . \\
H_{Y} & \text { for } Y \geq y_{M}
\end{array} .\right.
$$

The quantities $y_{m}$ and $y_{M}$ play the role of largest loss that results in the minimum premium $G_{Y}$ and of smallest loss that results in the maximum premium $H_{Y}$, respectively (see [8]). They are related to the rating parameters in this way:

$$
G_{Y}=\left(B_{Y}+L y_{m}\right) T \quad H_{Y}=\left(B_{Y}+L y_{M}\right) T
$$

so that the retrospective premium can be restated as

$$
\Pi(Y)=G_{Y}+L T\left[\min \left(Y, y_{M}\right)-\min \left(Y, y_{m}\right)\right]
$$

Note that from (3), in order to have $y_{m} \geq 0$ (and $y_{M} \geq 0$ ), necessarily it is $G_{Y} \geq B T$.

\section{Expected Retrospective Premium}

We analyze the expected retrospective premium by referring to the limited expected value function (l.e.v.f.) $M_{Y}$ :

$$
M_{Y}(u)=E[\min (Y, u)]=\int_{0}^{u}\left(1-F_{Y}(y)\right) d y, \quad(u \geq 0)
$$

where $F_{Y}$ denotes the distribution function di $Y$. The expected retrospective premium $E[\Pi(Y)]$ can be written as

$$
E[\Pi(Y)]=G_{Y}+L T\left(M_{Y}\left(y_{M}\right)-M_{Y}\left(y_{m}\right)\right) .
$$

Given the basic premium $B_{Y}$ and the rating parameters $L$ and $T$, in this paper we study the expected premium (6) as a function $\Psi\left(y_{m}, y_{M}\right)$ of $y_{m}$ and $y_{M}$ :

$$
\Psi\left(y_{m}, y_{M}\right)=B_{Y} T+L T\left(y_{m}+\int_{y_{m}}^{y_{M}}\left(1-F_{Y}(y)\right) d y\right)
$$


Observe that the inequality $0 \leq \int_{y_{m}}^{y_{M}}\left(1-F_{Y}(y)\right) d y \leq y_{M}-y_{m}$ and (3) imply

$$
G_{Y}=B_{Y} T+L T y_{m} \leq \Psi\left(y_{m}, y_{M}\right) \leq B_{Y} T+L T y_{M}=H_{Y}
$$

where the minimum and maximum premia, $G_{Y}$ and $H_{Y}$ respectively, are image values of $\Psi: G_{Y}=\Psi\left(y_{m}, y_{m}\right)$ and $H_{Y}=\Psi\left(y_{M}, y_{M}\right)$. The particular case of a minimum premium proportional to the basic premium through $T$ (i.e. $\left.G_{Y}=B_{Y} T\right)$, corresponds to

$$
\Psi\left(0, y_{M}\right)=B_{Y} T+L T \int_{0}^{y_{M}}\left(1-F_{Y}(y)\right) d y
$$

Moreover, given that $\lim _{u \rightarrow \infty} M_{Y}(u)=E[Y]$, it is

$$
\lim _{y_{M} \rightarrow \infty} \Psi\left(y_{m}, y_{M}\right)=B_{Y} T+L T\left(y_{m}+E[Y]-M_{Y}\left(y_{m}\right)\right)
$$

where $E[Y]-M_{Y}\left(y_{m}\right)=E\left[\max \left(Y-y_{m}, 0\right)\right]$.

If we assume that the distribution function $F_{Y}$ is continuous, then the function $\Psi\left(y_{m}, y_{M}\right)$ turns out to be differentiable with

$$
\frac{\partial}{\partial y_{m}} \Psi\left(y_{m}, y_{M}\right)=L T F_{Y}\left(y_{m}\right) \quad \frac{\partial}{\partial y_{M}} \Psi\left(y_{m}, y_{M}\right)=L T\left(1-F_{Y}\left(y_{M}\right)\right) .
$$

Moreover, $\Psi\left(y_{m}, y_{M}\right)$ results to be increasing as a function of the variable $y_{m}$ or as a function of $y_{M}$.

The difference between the expected premium $\Psi\left(y_{m}, y_{M}\right)$ and the minimum premium $G_{Y}=\Psi\left(y_{m}, y_{m}\right)$ is

$$
\Psi\left(y_{m}, y_{M}\right)-B_{Y} T-L T y_{m}=L T \int_{y_{m}}^{y_{M}}\left(1-F_{Y}(y)\right) d y
$$

it is a decreasing function of $y_{m}$, given $y_{M}$ (i.e., the maximum premium $H_{Y}$ ); it is an increasing function of $y_{M}$, given $y_{m}$ (i.e., the minimum premium $G_{Y}$ ). Moreover, this difference is bounded from above: in fact, given $y_{m}$, the limit (10) and the monotonic behaviour ensure the inequality

$$
\Psi\left(y_{m}, y_{M}\right)-B_{Y} T-L T y_{m} \leq L T\left(E[Y]-M_{Y}\left(y_{m}\right)\right) .
$$

The difference between the maximum premium $H_{Y}=\Psi\left(y_{M}, y_{M}\right)$ and the expected premium $\Psi\left(y_{m}, y_{M}\right)$ is

$$
B_{Y} T+L T y_{M}-\Psi\left(y_{m}, y_{M}\right)=L T\left(y_{M}-y_{m}-\int_{y_{m}}^{y_{M}}\left(1-F_{Y}(y)\right) d y\right)
$$

given $y_{m}$, it turns out to be an increasing function of $y_{M}$, while given $y_{M}$, it is a decreasing function of $y_{m}$ (by (11)). The difference is bounded from above, given $y_{M}$ : by decreasing monotonicity and setting $y_{m}=0$ it is

$$
B_{Y} T+L T y_{M}-\Psi\left(y_{m}, y_{M}\right) \leq L T\left(y_{M}-M_{Y}\left(y_{M}\right)\right) \text {. }
$$


The expected retrospective premium $\Psi\left(y_{m}, y_{M}\right)$ admits another representation by referring to the inverse function ${ }^{1} F_{Y}^{-1}$ :

$$
F_{Y}^{-1}(p)=\inf \left\{x \in \mathfrak{R} \mid F_{Y}(x) \geq p\right\}=\sup \left\{x \in \mathfrak{R} \mid F_{Y}(x)<p\right\} .
$$

The expected retrospective premium $\Psi\left(y_{m}, y_{M}\right)$ admits the following equivalent characterization

$$
\Psi\left(y_{m}, y_{M}\right)=\left\{\begin{array}{ll}
B_{Y} T+L T\left(y_{m}+M_{Y}\left(y_{M}\right)-M_{Y}\left(y_{m}\right)\right) & \text { for } y_{m}<F_{Y}^{-1}(1) \\
B_{Y} T+L T y_{m} & \text { for } y_{m} \geq F_{Y}^{-1}(1)
\end{array} .\right.
$$

Note, in fact, that if $y_{m} \geq F_{Y}^{-1}(1)$, then $M_{Y}\left(y_{m}\right)=M_{Y}\left(y_{M}\right)=E[Y]$.

\section{A numerical example}

To study the distribution of the random loss $Y$ we refer to the collective risk model (see [4]). Let $\mu_{Y}, \sigma_{Y}^{2}$ and $\gamma_{Y}$ denote the mean, variance and coefficient of skewness of $Y$, respectively. We assume $Y$ has the same distribution of the random variable $Z+z_{0}$, where $z_{0}$ is constant and $Z$ follows a gamma distribution with positive parameters $\alpha$ e $\beta$ :

$$
F_{Z}(z)=\frac{1}{\Gamma(\alpha)} \int_{0}^{z} x^{\alpha-1} \beta^{\alpha} e^{-\beta x} d x, \quad z \geq 0
$$

As it is well known, $F_{Z}(z)=\Gamma(\alpha ; \beta z)$ where $\Gamma(\alpha ; \beta z)$ is the incomplete gamma function, i.e.

$$
\Gamma(\alpha ; \beta z)=\frac{1}{\Gamma(\alpha)} \int_{0}^{\beta z} x^{\alpha-1} e^{-x} d x
$$

The parameters $\alpha, \beta$ and $z_{0}$ are chosen so that $Z+z_{0}$ and $Y$ have the same first three moments: $\mu_{Y}=z_{0}+\frac{\alpha}{\beta}, \sigma_{Y}^{2}=\frac{\alpha}{\beta^{2}}, \gamma_{Y}=\frac{2}{\sqrt{\alpha}}$, therefore the parameters are linked to $\mu_{Y}, \sigma_{Y}^{2}$ and $\gamma_{Y}$ according to the rules:

$$
\alpha=\frac{4}{\gamma_{Y}^{2}}, \quad \beta=\frac{2}{\gamma_{Y} \sigma_{Y}} \quad \text { and } \quad z_{0}=\mu_{Y}-\frac{2 \sigma_{Y}}{\gamma_{Y}} .
$$

The l.e.v.f. $M_{Z}$ of $Z$ is a continuous increasing function (see [6])

$$
M_{Z}(u)=\frac{\alpha}{\beta} \Gamma(\alpha+1 ; \beta u)+u[1-\Gamma(\alpha ; \beta u)], \quad u \geq 0
$$

with first derivative $M_{Z}^{\prime}(u)=1-\Gamma(\alpha ; \beta u)$. Furthermore, given the assumptions on $Y$ and $Z$ and the properties of the l.e.v.f., we obtain the following results:

$$
M_{Y}(u)=M_{Z+z_{0}}(u)=M_{Z}\left(u-z_{0}\right)+z_{0}, \quad u \geq z_{0}
$$

\footnotetext{
${ }^{1} p \in[0,1]$, and, by convention, inf $\varnothing=+\infty$
} 


$$
\lim _{u \rightarrow z_{0}} M_{Y}(u)=M_{Y}\left(z_{0}\right)=z_{0} \quad \lim _{u \rightarrow \infty} M_{Y}(u)=\frac{\alpha}{\beta}+z_{0}
$$

Therefore, referring to (7) and (22), the expected retrospective premium $\Psi\left(y_{m}, y_{M}\right)$ admits the following formulation:

$$
\Psi\left(y_{m}, y_{M}\right)=B_{Y} T+L T\left(y_{m}+M_{Z}\left(y_{M}-z_{0}\right)-M_{Z}\left(y_{m}-z_{0}\right)\right)
$$

where $y_{M} \geq y_{m} \geq z_{0}$ and

$$
\begin{aligned}
M_{Z}\left(y_{M}-z_{0}\right)-M_{Z}\left(y_{m}-z_{0}\right)= & \frac{1}{\beta \Gamma(\alpha)} \int_{\beta\left(y_{m}-z_{0}\right)}^{\beta\left(y_{M}-z_{0}\right)} x^{\alpha} e^{-x} d x \\
& +y_{M}\left[1-\Gamma\left(\alpha ; \beta\left(y_{M}-z_{0}\right)\right]-y_{m}\left[1-\Gamma\left(\alpha ; \beta\left(y_{m}-z_{0}\right)\right]\right.\right. \\
& +\frac{z_{0}}{\Gamma(\alpha)} \int_{\beta\left(y_{m}-z_{0}\right)}^{\beta\left(y_{M}-z_{0}\right)} x^{\alpha-1} e^{-x} d x
\end{aligned}
$$

In this way, we obtain the following relations:

i) $\Psi\left(z_{0}, y_{M}\right)=B_{Y} T+L T\left(z_{0}+M_{Z}\left(y_{M}-z_{0}\right)\right)$

ii) $\Psi\left(y_{m}, z_{0}\right)=\Psi\left(z_{0}, z_{0}\right)=B_{Y} T+L T z_{0}$

iii) $\lim _{y_{m} \rightarrow y_{M}} \Psi\left(y_{m}, y_{M}\right)=B_{Y} T+L T y_{M}$

iv) $\lim _{y_{M} \rightarrow \infty} \Psi\left(y_{m}, y_{M}\right)=B_{Y} T+L T\left(y_{m}+\frac{\alpha}{\beta}-M_{Z}\left(y_{m}-z_{0}\right)\right)$

As we have seen, the differences between the expected retrospective premium $\Psi\left(y_{m}, y_{M}\right)$ and the extreme premia (i.e., $G_{Y}$ and $\left.H_{Y}\right)$ are bounded. In this case, by (13) the difference $\Psi\left(y_{m}, y_{M}\right)-G_{Y}$ is bounded from above

$$
\Psi\left(y_{m}, y_{M}\right)-B_{Y} T-L T y_{m} \leq L T\left(\frac{\alpha}{\beta}-M_{Z}\left(y_{m}-z_{0}\right)\right)
$$

and by $(15)$ the difference $H_{Y}-\Psi\left(y_{m}, y_{M}\right)$ is bounded from above

$$
B_{Y}+L T y_{M}-\Psi\left(y_{m}, y_{M}\right) \leq L T\left(y_{M}-z_{0}-M_{Z}\left(y_{M}-z_{0}\right)\right)
$$

In Figures 1 and 2, the graphs of the function $\Psi\left(y_{m}, y_{M}\right)$ and of some level curves are plotted ${ }^{2}$, with parameters $\alpha=4, \beta=0.004, B_{Y}=400, T=1.002$, $L=0.9, z_{0}=9000$ and ranges $y_{m} \in[9050,10000], y_{M} \in[10000,11000]$. Note that some of the properties discussed in Section 3 are highlighted in the images.

\footnotetext{
${ }^{2}$ Wolfram Research, Inc., Mathematica, Version 12.3, Champaign, IL (2021).
} 


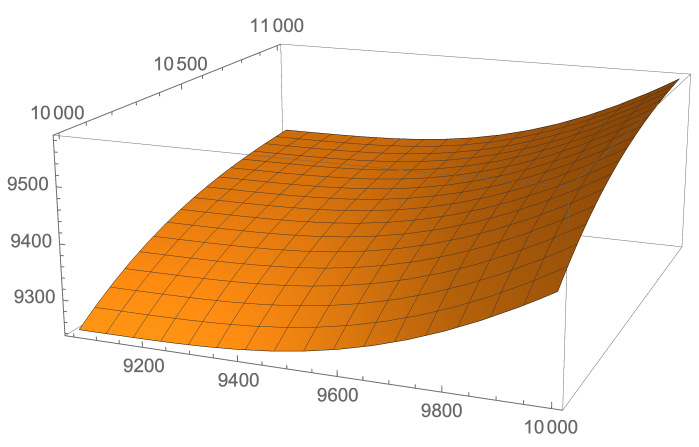

Figure 1: $\Psi\left(y_{m}, y_{M}\right)$

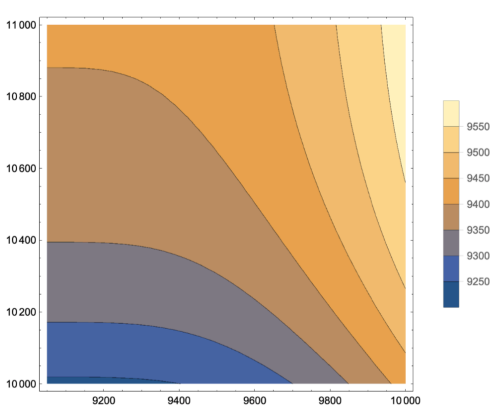

Figure 2: Level curves of $\Psi\left(y_{m}, y_{M}\right)$

\section{Concluding remarks}

The retrospective rating plan is a particular kind of risk-sharing agreement evolving over time, where the insurance premium is a function of the actual loss paid under the contract. Knowing the expected value of the premium is important in implementing a retrospective rating plan for both the insurer and the policyholder, so that they can plan their business.

In this paper we have analyzed some properties of the expected retrospective premium that can offer possible interpretations and practical applications. For example, by setting the initial values $\bar{y}_{m}$ and $\bar{y}_{M}$ in order to guarantee an initial expected premium $\Psi\left(\bar{y}_{m}, \bar{y}_{M}\right)$, upon renewal of the contract the parameters may be subject to changes. Thanks to (11), the corresponding new value of the expected premium $\Psi\left(y_{m}, y_{M}\right)$ can be approximated around the point $\left(\bar{y}_{m}, \bar{y}_{M}\right)$ :

$$
\begin{aligned}
\Psi\left(y_{m}, y_{M}\right) \approx & \Psi\left(\bar{y}_{m}, \bar{y}_{M}\right)+\operatorname{LTF} F_{Y}\left(\bar{y}_{m}\right)\left(y_{m}-\bar{y}_{m}\right) \\
& +L T\left(1-F_{Y}\left(\bar{y}_{M}\right)\right)\left(y_{M}-\bar{y}_{M}\right) .
\end{aligned}
$$

Note that (27) just requires the knowledge (or estimate) of the values $F_{Y}\left(\bar{y}_{m}\right)$ and $F_{Y}\left(\bar{y}_{M}\right)$.

Following [1] and [2], the topic here studied can be also further investigated under different assumptions.

\section{References}

[1] J. Cai, Y. Chi, Optimal reinsurance designs based on risk measures: A review, Statistical Theory and Related Fields, 4 (1) (2020), 1-13.

https://doi.org/10.1080/24754269.2020.1758500 
[2] A. Campana, P. Ferretti, On optimal reinsurance with stochastic premium, Applied Mathematical Sciences, 13 (18) (2019), 859-867.

https://doi.org/10.12988/ams.2019.97102

[3] X. Chen, Y. Chi, K.S. Tan, The design of an optimal retrospective rating plan, Astin Bulletin, 46 (1) (2016), 141-163.

https://doi.org/10.1017/asb.2015.19

[4] C.D. Daykin, T. Pentikinen, M. Pesonen, Practical risk theory for actuaries, Chapman and Hall/CRC, 1993.

https://doi.org/10.1201/9781482289046

[5] W.R. Gillam, Retrospective rating: excess loss factors, in: Proceedings of the Casualty Actuarial Society, LXXVIII (1991), 1-40.

http://www.casact.org/pubs/proceed/proceed91/91001.pdf

[6] S.A. Klugman, H.H. Panjer, G.E. Willmot, Loss Models: From Data to Decisions, J. Wiley \& Sons, 2019. https://doi.org/10.1002/9780470391341

[7] G.G. Meyers, An analysis of retrospective rating, in: Proceedings of the Casualty Actuarial Society, LXVII (1980), 323-354.

[8] G.G. Meyers, Retrospective premium, in Encyclopedia of Actuarial Science, (ed. J. Teugels and B. Sundt), John Wiley \& Sons, Chichester, 3 (2004), 1452-1456.

[9] J.F. Walhin, L. Herfurth, P. de Longueville, The practical pricing of Excess of Loss treaties: actuarial, financial, economic and commercial aspects. Belgian Actuarial Bulletin, 31 (2001), 1-17.

\section{Received: July 1, 2021; Published: July 14, 2021}

